\title{
MEG3 Suppresses Human Pancreatic Neuroendocrine Tumor Cells Growth and Metastasis by Down-Regulation of Mir-183
}

\author{
Yuan-Yuan Zhang $^{\text {a }}$ Hao-Miao Feng ${ }^{b}$ \\ aDepartment of Endocrinology, Eastern Medical District of Linyi People's Hospital, Linyi, \\ bDepartment of General Surgery, Linyi People's Hospital, Linyi, China
}

\section{Key Words}

Lncrna MEG3 • Pancreatic neuroendocrine tumors (pNETs) • BON1 cells • MiR-183 • BRI3 • P38/ERK/AKT and Wnt/ $\beta$-Catenin signaling

\begin{abstract}
Background/Aims: Pancreatic neuroendocrine tumors (pNETs) are rare neoplasms which arise from pancreatic islet cells. Recently, IncRNA MEG3 has been reported as a tumor suppressor in variety cancers. This study aimed to reveal the functional effects of MEG3 on pNETs which has not been uncovered previously. Methods: The expression of MEG3, miR183, and BRI3 in BON1 cells were altered by transfection with their specific vectors/shRNA, or mimic/inhibitor. Thereafter, cell viability, apoptosis, the protein expressions of cell cycle related factors, and apoptosis associated factors, as well as cell migration and invasion were respectively assessed by typan blue staining, flow cytometry, western blotting, and transwell assay. Results: MEG3 was low expressed in BON1 and QGP-1 cells, when compared to three normal cell lines (HEK293, CCL-153, and EC-304). MEG3 overexpression decreased BON1 cells viability, invasion, migration, but significantly induced apoptosis. miR-183 was a direct target of MEG3, and miR-183 up-regulation abolished the anti-growth and anti-metastasis effects of MEG3 overexpression on BON1 cells. Moreover, BRI3 was a target of miR-183, and BRI3 exhibited a tumor-promoting role possibly via activation of p38/ERK/AKT and Wnt/ $/$-Catenin signaling in BON1 cells. Conclusion: This study demonstrated a tumor suppressive effect of MEG3 in BON1 cells that suppresses tumor cells growth and metastasis. A novel regulatory mechanism has been revealed that modulation of MEG3/miR-183/BRI3 axis may be pivotal in pNET.

\section{Introduction}

Pancreatic neuroendocrine tumors (pNETs) are a captivating and diverse group of rare neoplasms, which arise from pancreatic islet cells [1]. pNETs comprise only $1 \%$ to $2 \%$ of all pancreatic neoplasms, and given the rarity of this tumor, well-designed randomized controlled trials on surgical treatment for pNET are not available [2, 3]. In addition to surgical, 
patient with advanced or metastatic pNETs requires a multidisciplinary approach, including targeted therapies, peptide receptor radiotherapy, local ablation and interventional therapy [3]. However, there are still many unanswered questions about treatment of this tumor, which need an improved understanding of the mechanisms underlying tumor cells inhibition.

Long non-coding RNAs (lncRNAs) are one kind of endogenous RNA comprises a sequence larger than 200 nucleotides but with no significant or functional open reading frame(s) [4]. Since their discoveries, a large amount of IncRNA has been found being dysregulated in a range of cancers [5]. In addition, they are being identified and characterized for serial steps of cancer development, including tumor initiation, growth, and metastasis [6]. Maternally expressed gene 3 (MEG3), a lncRNA that has attracted much research interest, is aberrantly expressed in several human cancers including ovarian cancer [7], gastric cancer [8], colorectal cancer [9], cervical cancer [10], and malignant glioma [11]. Moreover, according to these previous studies, MEG3 exhibited anti-cancer effects via regulation of cell proliferation, apoptosis, and metastasis. However, to date whether MEG3 also plays a role in pNETs remains unclear.

microRNAs (miRNAs) are a class of short non-coding RNAs, that regulate the expression of target genes by interaction with the 3'-un-translated region (3'-UTR) of their mRNAs, resulting in translation inhibition or mRNA degradation [12]. In the cancer studies, miRNAs can function to be oncogenic or tumor-suppressive [13]. Besides, an increasing study have evidenced that IncRNAs can act as a competing endogenous RNA (ceRNA) via sponging miRNAs $[13,14]$. In previous studies, MEG3 has been described to be a ceRNA that regulate miR-21 [15, 16], miR-1297 [17], and miR-140-5p [18]. Thus, we speculated that MEG3 may serve as a ceRNA which connecting some other miRNAs in pNETs.

This study aimed to reveal the functional effects of MEG3 on pNETs, and tried to explore its underlying mechanism(s). pNET cell line BON1 were used in this study, and the expression of MEG3, miR-183, and BRI3 in cells were altered by transfection. The effects of ectopic expression of MEG3, miR-183, and BRI3 on BON1 cells were respectively assessed by detection of cell growth and metastasis. This study revealed a novel regulatory mechanism in pNET cells, and may provide a new insight in the therapy of pNET.

\section{Materials and Methods}

\section{Cell culture}

Human kidney epithelial cell line HEK293, human lung fibroblast CCL-153, and human endothelial cell line EC-304 were all purchased from the American Type Culture Collection (ATCC, Manassas, VA). BON1 cell line was a gift from Dr. He Na, Department of Gastroenterology, the First Affiliated Hospital with Nanjing Medical University (Nanjing, China) [19]. QGP-1 cells were purchased from the Japanese Collection of Research Bioresources Cell Bank (JRCB, Osaka, Japan). QGP-1 cells were grown in RPMI-1640 medium (Invitrogen, Carlsbad, CA) supplemented with 10\% fetal bovine serum (FBS, Invitrogen). All other cell lines were cultured in Dulbecco's modified Eagle's medium (DMEM, Invitrogen) supplemented with 10\% FBS. All cells were maintained at $37^{\circ} \mathrm{C}$ in $5 \% \mathrm{CO}_{2}$. Cells in logarithmic phase were used for throughout this work.

\section{qRT-PCR}

Total RNA was isolated from cells by using Trizol reagent (Invitrogen) according to the manufacturer's instructions. cDNA of MEG3 and BRI3 was synthesized with PrimeScript RT reagent Kit (Promega, Madison, WI, USA). qRT-PCR was carried out by using TaqMan Power SYBR Green PCR Mix (Invitrogen). For the test of miR-183, the Taqman MicroRNA Reverse Transcription Kit (Applied Biosystems, Foster City, CA) was used for reverse transcription. The miR-183 level was quantified by qRT-PCR using the Taqman Universal Master Mix II (Applied Biosystems, Foster City, CA). All amplification reactions were performed by using an ABI PRISM 7500 Sequence Detection System (Applied Biosystems). Data were calculated by the $2^{-\Delta \Delta C}$ method [20]. The internal control genes were GAPDH for MEG3 and BRI3, and U6 snRNA for miR-183.

Transfection

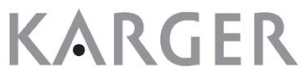




\section{Cellular Physiology Cell Physiol Biochem 2017;44:345-356 \begin{tabular}{l|l|l} 
and BOI: 10.1159/000484906 & $\begin{array}{l}\text { C } 2017 \text { The Author(s). Published by S. Karger AG, Basel } \\
\text { www.karger.com/cpb }\end{array}$
\end{tabular} \\ Zhang/Feng: the Role of MEG3 in Pancreatic Neuroendocrine Tumor}

The sequences of MEG3 were ligated into the pEX-2 vector (Genepharma, Shanghai, China), and used as an MEG3 expressing vector; empty pEX-2 vector was used as its negative control (NC). miR-183 mimic, miR-183 inhibitor, and their NCs (mimic NC and inhibitor NC) were purchased from Ribobio (Guangzhou, China). For BRI3, the BRI3 mRNA sequences were synthesized and subcloned into the pEX-2 vector, and the empty pEX-2 vector served as NC (Genepharma). shRNA targeted BRI3 was constructed in U6/GFP/ Neo plasmids, and non-target sequence served as its NC (GenePharma). All transfections were performed by the lipofectamine 3000 reagent (Invitrogen) according to the manufacturer's instructions. After $48 \mathrm{~h}$ of transfection, the cells were collected for use in the further investigations. Transfected cells were selected by $0.5 \mathrm{mg} / \mathrm{mL}$ G418 (Sigma-Aldrich, St Louis, M0) to become stably transfected cells.

\section{Cell viability assay}

Cell viability was measured by using a Typan Blue Staining Cell Viability Assay Kit (Beyotime, Shanghai, China). After transfection and a $48 \mathrm{~h}$-incubation in the fresh medium, $5 \times 10^{3}$ BON1 cells were collected and resuspended in $1 \mathrm{~mL}$ Cell Resuspension Solution. Then, $1 \mathrm{~mL}$ Trypan Blue Solution was added into each sample and staining for $5 \mathrm{~min}$ at room temperature. Cells were counted using a hemocytometer and the ratio of living/total cells was determined.

\section{Apoptosis assay}

Cell apoptosis analysis was performed using Annexin V-FITC Apoptosis Detection Kit (Beyotime). Briefly, after $48 \mathrm{~h}$ of incubation at $37^{\circ} \mathrm{C}, 1 \times 10^{5}$ transfected cells were collected and resuspended in 195 $\mu \mathrm{L}$ Annexin V-FITC Binding Buffer. Then, $5 \mu \mathrm{L}$ Annexin V-FITC and $10 \mu \mathrm{L}$ PI were added, and the samples were incubated in the dark at room temperature for $20 \mathrm{~min}$. Flow cytometry analysis was done by using a FACS can (Beckman Coulter, Fullerton, CA), and data were analyzed by using Flowjo software (Treestar, San Carlos, CA).

\section{Transwell assay}

Cell migration and invasion were determined by using a modified two-chamber (Costar-Corning, New York, USA), with a pore size of $8 \mathrm{~mm}$. For migration assay, the transfected cells suspended in $200 \mathrm{~mL}$ of serum-free medium were seeded on the upper compartment, and $600 \mathrm{~mL}$ of complete medium was added to the lower compartment. After $48 \mathrm{~h}$ of incubation at $37^{\circ} \mathrm{C}$, non-traversed cells in the upper chamber were removed by a cotton swab. Traversed cells on the lower side of the filter were stained with $0.1 \%$ crystal violet (Sigma-Aldrich), and counted under the microscope. The invasive behavior of cells was detected the same as migration assay, except the filters were pre-coated with Matrigel membrane (BD Biosciences, San Jose, CA).

\section{Dual-luciferase reporter assay}

The fragments from MEG3 and BRI3 containing the predicted miR-183 binding site was amplified by PCR and then cloned into a pmirGlO Dual-luciferase miRNA Target Expression Vector (Promega, Madison, WI) to form the reporter vector MEG3-wild-type (MEG3-wt) and BRI3-wild-type (BRI3-wt). To mutate the putative binding site of miR-183 in the MEG3 and BRI3, the sequences of putative binding site was replaced and were named as MEG3-mutated-type (MEG3-mt) and BRI3-mutated-type (BRI3-mt). These four vectors were respectively co-transfected with miR-183 mimic or mimic control into BON1 cells by using the lipofectamine 3000 reagent (Invitrogen). Dual-Luciferase Reporter Assay System (Promega, Madison, WI) were used for testing the luciferase activity.

Western blot

Cellular protein was extracted using RIPA lysis buffer (Beyotime), and quantified using the BCA ${ }^{\mathrm{TM}}$ Protein Assay Kit (Pierce, Appleton, WI). Equivalent amounts of whole-cell extracts from each sample were resolved over 10\%-12\% sodium dodecyl sulfate-polyacrylamide gel electrophoresis (SDS-PAGE) and transferred to polyvinylidene difluoride (PVDF) membranes. The membranes were blocked within 5\% nonfat milk for $1 \mathrm{~h}$ at room temperature and then incubated in primary antibodies for the specific detection of PCNA (ab18197, Abcam, Cambridge, MA), Cyclin E1 (ab3927), CDK2 (ab32147), Cyclin D1 (ab16663), CDK4 (ab68266), Bcl-2 (ab194583), Bax (ab32503), caspase-3 (ab13586), caspase-9 (ab184786), BRI3 (ab69578), Wnt3a (ab28472), Wnt5a (ab72583), $\beta$-Catenin (ab32572), and p-p38MAPK (\#4092, Cell 


\section{Cellular Physiology Cell Physiol Biochem 2017;44:345-356 \begin{tabular}{l|l|l} 
and Biochemistry & DOI: 10.1159/000484906 & $\begin{array}{l}\text { (c) } 2017 \text { The Author(s). Published by S. Karger AG, Basel } \\
\text { www.karger.com/cpb }\end{array}$
\end{tabular}

Signaling Technology Inc., Danvers, MA), p38MAPK (\#14451), p-ERK (\#9106), ERK (\#5013), p-AKT (\#4060), AKT (\#14702), GAPDH (\#5174). After washing in PBS, the membranes were incubated with the rabbit anti-mouse (\#58802) and mouse anti-rabbit (\#14708) IgG secondary antibodies at room temperature for $1 \mathrm{~h}$. Positive signals were developed by EasyBlot ECL Kit (Sangon Biotech, Shanghai, China).

\section{Statistical analysis}

Data represent the mean \pm standard deviation (SD) of three independent experiments. Statistical analyses were performed by SPSS 20 (IBM, New York, NY) using a one-way analysis of variance (ANOVA). $P$-value of $<0.05$ was considered to indicate a statistically significant result.

\section{Results}

MEG3 was low expressed in BON1 cells

Firstly, we detected the expression level of MEG3 in human kidney epithelial cell line HEK293, human lung fibroblast CCL-153, human endothelial cell line EC-

304, and human pNET cell lines BON1 and QGP-1. qRT-PCR analysis results given in Fig. 1
showed that, the levels of MEG3 in BON1 and QGP-1 cells were much lower than those in other three normal cell lines $(P<0.05$ or $P<0.01)$.

\section{MEG3 suppressed BON1 cells growth and metastasis}

To detect whether MEG3 play a role in pNET, the expression of MEG3 in BON1 cells was overexpressed by transfection with its expressing vector (pEX-MEG3). As a result, MEG3 level was significantly up-regulated $(P<0.001$, Fig. 2A) after $48 \mathrm{~h}$ of transfection. MEG3 significantly reduced cell viability $(P<0.05$, Fig. $2 \mathrm{~B})$, but induced apoptosis $(P<0.01$, Fig. 2C). Western blotting analysis results showed that, cell cycle correlated proteins including PCNA, Cyclin E1, CDK1, Cyclin D1, and CDK4 were all down-regulated after MEG3 was overexpressed (Fig. 2D). Besides, pro-apoptosis proteins including Bax, cleaved caspase-3 and cleaved caspase- 9 were remarkably up-regulated by MEG3 overexpression, while antiapoptosis protein Bcl-2 was down-regulated (Fig. 2E). Moreover, transwell assay results showed that MEG3 significantly diminished both cell migration and invasion (both $P<0.05$, Fig. 2F-2G). Altogether, these data suggested an anti-growth and anti-metastasis role of MEG3 in BON1 cells.

miR-183 was a direct target of MEG3

An increasing literature has evidenced that lncRNA can act as a ceRNA via sponging miRNA $[13,14]$. Herein, we detected the cross-regulation between MEG3 and miR-183, since in a recent review, miR-183 has been reported as a pivotal miRNA in endocrine cancer metastasis [21]. Firstly, we detected the expression of miR-183 in human kidney epithelial cell line HEK293 and human pNET cell lines BON1 and QGP-1. qRT-PCR analytical results showed that expression levels of miR-183 in BON1 and QGP-1 cells were significantly higher than those in HEK293 cells $(P<0.01$ or $P<0.001$, Fig. 3A). Then, we found that the expression of miR-183 was significantly down-regulated after MEG3 was overexpressed $(P<$ 0.001, Fig. 3B). Additionally, the luciferase activity was significantly reduced after cells were 


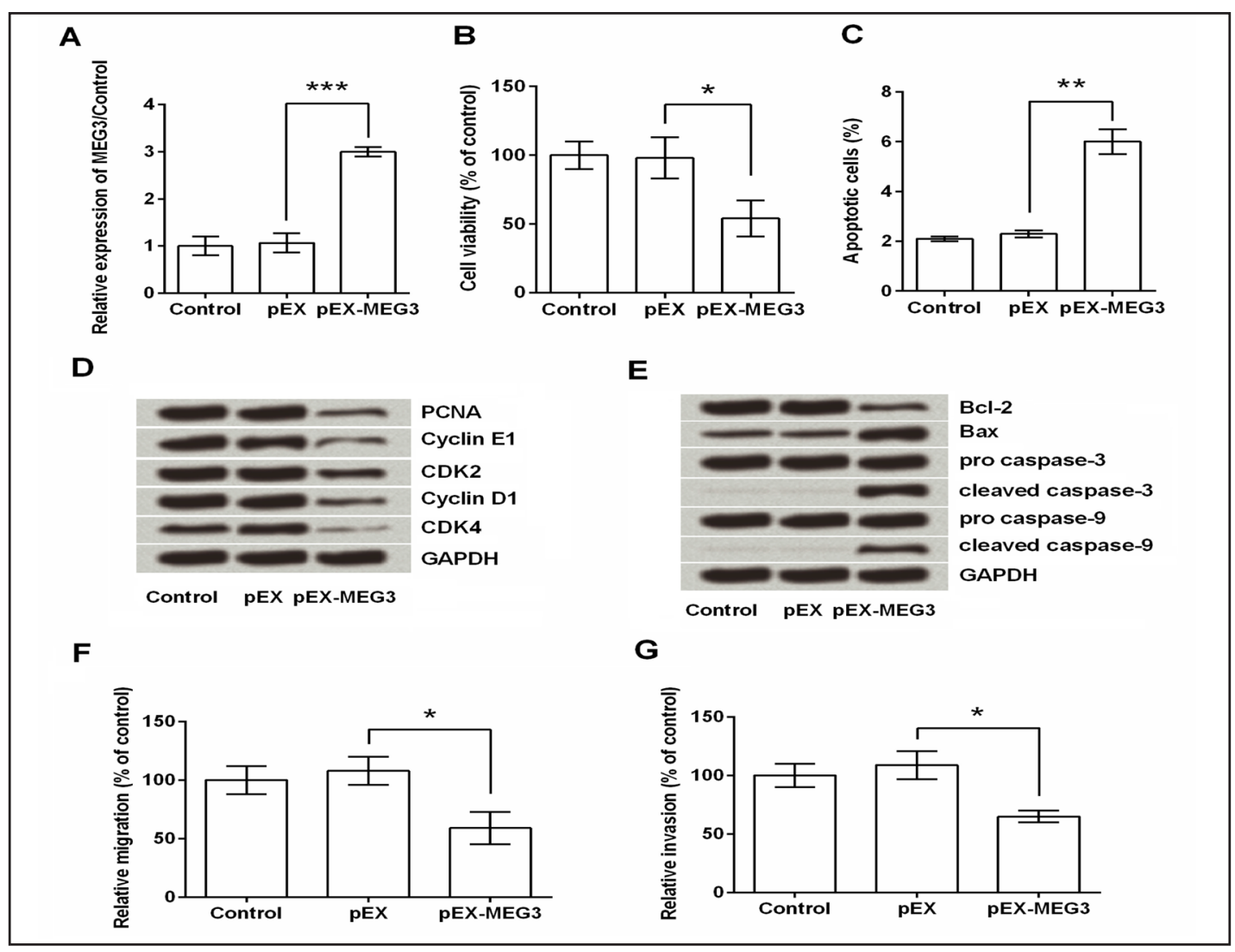

Fig. 2. MEG3 suppressed BON1 cells growth and metastasis. BON1 cells were transfected with MEG3 expressing vector (pEX-MEG3) or its negative control ( $\mathrm{pEX}$ ) for $48 \mathrm{~h}$. (A) The expression of MEG3 in cells was detected by qRT-PCR. After another $48 \mathrm{~h}$ of incubation in the fresh medium, (B) cell viability, (C) apoptotic cells rate, (D) the protein expression of cell cycle related factors, (E) the protein expression of apoptosis associated factors, (F) cell migration, and (G) cell invasion were respectively assessed by typan blue staining, flow cytometry, western blotting, and transwell assay. Data represent the mean \pm SD of three independent experiments. ${ }^{*} \mathrm{P}<0.05$, ${ }^{* *} \mathrm{P}<0.01$, and ${ }^{* *} \mathrm{P}<0.01$ (ANOVA).

co-transfected with miR-183 mimic and MEG3-wt $(P<0.01$, Fig. 3C). These data indicated that miR-183 was a direct target of MEG3, and it was negatively regulated by MEG3.

miR-183 up-regulation abolished the anti-tumor functions of MEG3 in BON1 cells

We then co-transfected MEG3 expressing vector and miR-183 mimic into BON1 cells to explore whether miR-183 was involved in the anti-tumor effects of MEG3. As results shown in Fig. 4A, the expression of miR-183 in BON1 cells was successfully overexpressed and suppressed after transfection with miR-183 mimic and miR-183 inhibitor (both $P<$ 0.001 ). More importantly, we found that miR-183 up-regulation abolished the anti-growth and anti-metastasis functions of MEG3, as cell viability was increased $(P<0.01$, Fig. 4B), apoptotic cells number was reduced $(P<0.001$, Fig. $4 \mathrm{C})$, cell cycle correlated proteins were up-regulated (Fig. 4D), pro-apoptosis proteins were down-regulated, anti-apoptosis protein was up-regulated (Fig. 4E), as well as migrated and invasive cells number were increased (both $P<0.01$, Fig. 4F-4G) by addition of miR-183 mimic.

\section{BRI3 was a direct target of miR-183}

A previous study has reported that BRI3 overexpression lead to the proliferation and migration of breast cancer cells [22]. This study we detected whether BRI3 is involved in miR183 modulated BON1 cells. We firstly detected the expression level of BRI3 in HEK293, BON1 
Fig. 3. miR-183 was a direct target of MEG3. (A) The expression level of miR-183 in human kidney epithelial cell line HEK293 and human pancreatic neuroendocrine tumor (pNET) cell line BON1 and QGP-1 were detected by qRT-PCR. (B) BON1 cells were transfected with MEG3 expressing vector ( $p E X-M E G 3$ ) or its negative control ( $\mathrm{pEX}$ ) for $48 \mathrm{~h}$. The expression of miR-183 in cells was then detected by qRT-PCR. (C) Dual-luciferase reporter assay was performed to detect whether miR-183 was a target of MEG3. Data represent the mean \pm SD of three independent experiments. ${ }^{* *}$ $\mathrm{P}<0.01$, and ${ }^{* *} \mathrm{P}<0.01$ (ANOVA).

and QGP-1 cells. qRT-PCR analytical results showed that expression levels of BRI3 in BON1 and QGP-1 cells were significantly lower than those in HEK293 cells $(P<0.001$, Fig. 5A). qRT-PCR and western blot analytical results showed that, the expression of BRI3 was down-regulated in cells which were transfected with miR183 inhibitor; in contrast, BRI3 was up-regulated in miR-183 mimic transfected cells $(P<0.01$, or $P<$ 0.001 , Fig. 5B-5C). Furthermore, the luciferase activity was significantly increased by co-transfection with miR-183 mimic and BRI3-wt $(P<$ 0.01 , Fig. 5D). These data suggested BRI3 was a direct target of miR-183, and it was positively regulated by

A

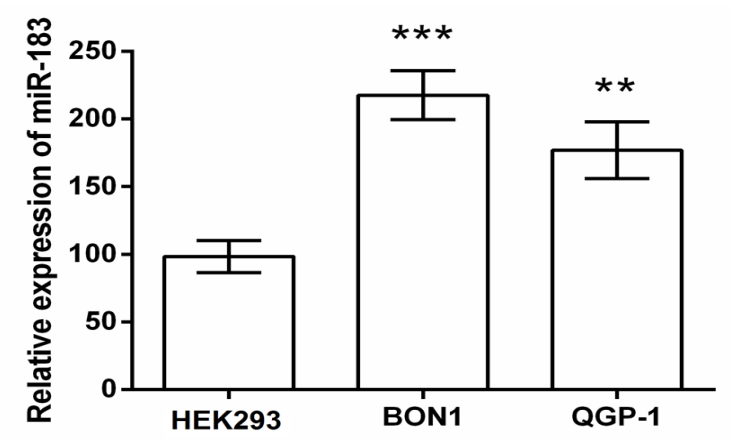

B
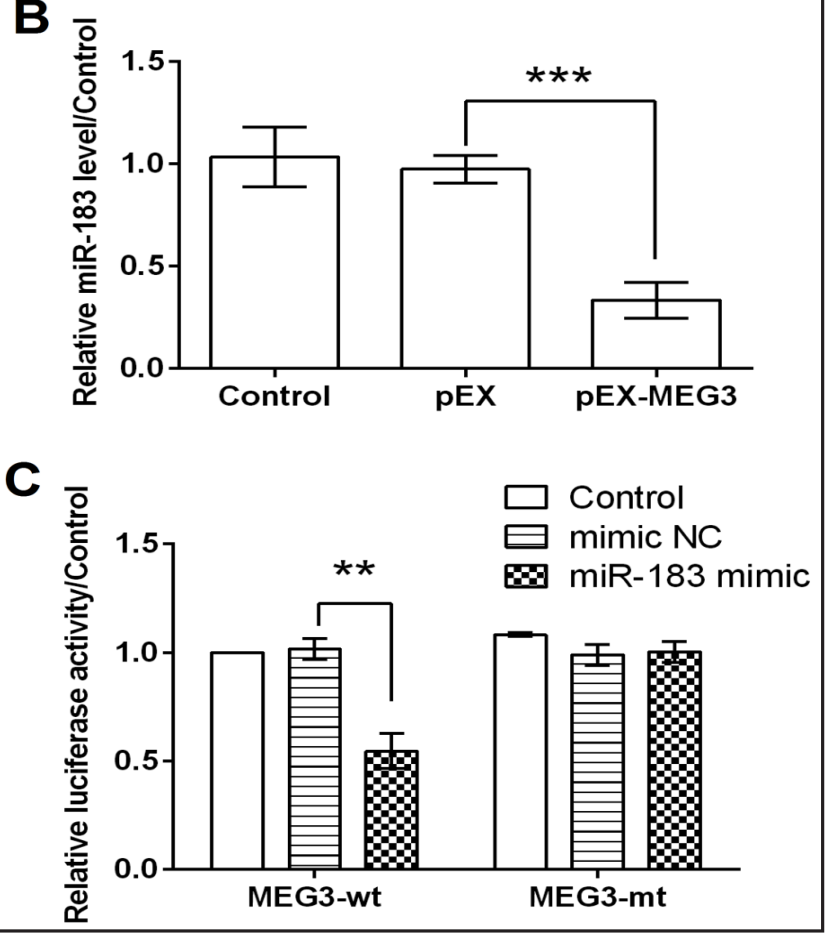
miR-183.

\section{BRI3 promoted BON1 cells growth and metastasis}

Next, the expression of BRI3 in BON1 cells was altered by transfection with its expressing vector or shRNA. As results shown in Fig. 6A, the mRNA level of BRI3 was up-regulated in cells transfected with pEX-BRI3, and was down-regulated in sh-BRI3 transfected cells (both $P<$ 0.001). Moreover, we found that BRI3 exerted the pro-growth and pro-metastasis functions on BON1 cells, as cell viability was improved $(P<0.001$, Fig. 6B), apoptotic cells rate was reduced $(P<0.05$, Fig. 6C), cell cycle correlated proteins were up-regulated (Fig. 6D), and migrated and invasive cells number were increased (both $P<0.001$, Fig. 6F-6G) by BRI3 overexpression. However, BRI3 silence showed the anti-growth and anti-metastasis role in BON1 cells $(P<0.01$, or $P<0.001$, Fig. 6B-6G).

\section{BRI3 activated $p 38 / E R K / A K T$ and Wnt/ $\beta$-Catenin signaling pathways}

Furthermore, we detected the expression levels of main factor in p38/ERK/AKT and Wnt $/ \beta$-Catenin signaling pathways, to further reveal via which mechanism(s) BRI3 promoted BON1 cells. As results shown in Fig. 7A-7B, up-regulations of p-p38MAPK, p-ERK, p-AKT, Wnt3a, Wnt5a, and $\beta$-Catenin were observed in BON1 overexpressing-cells. However, these 


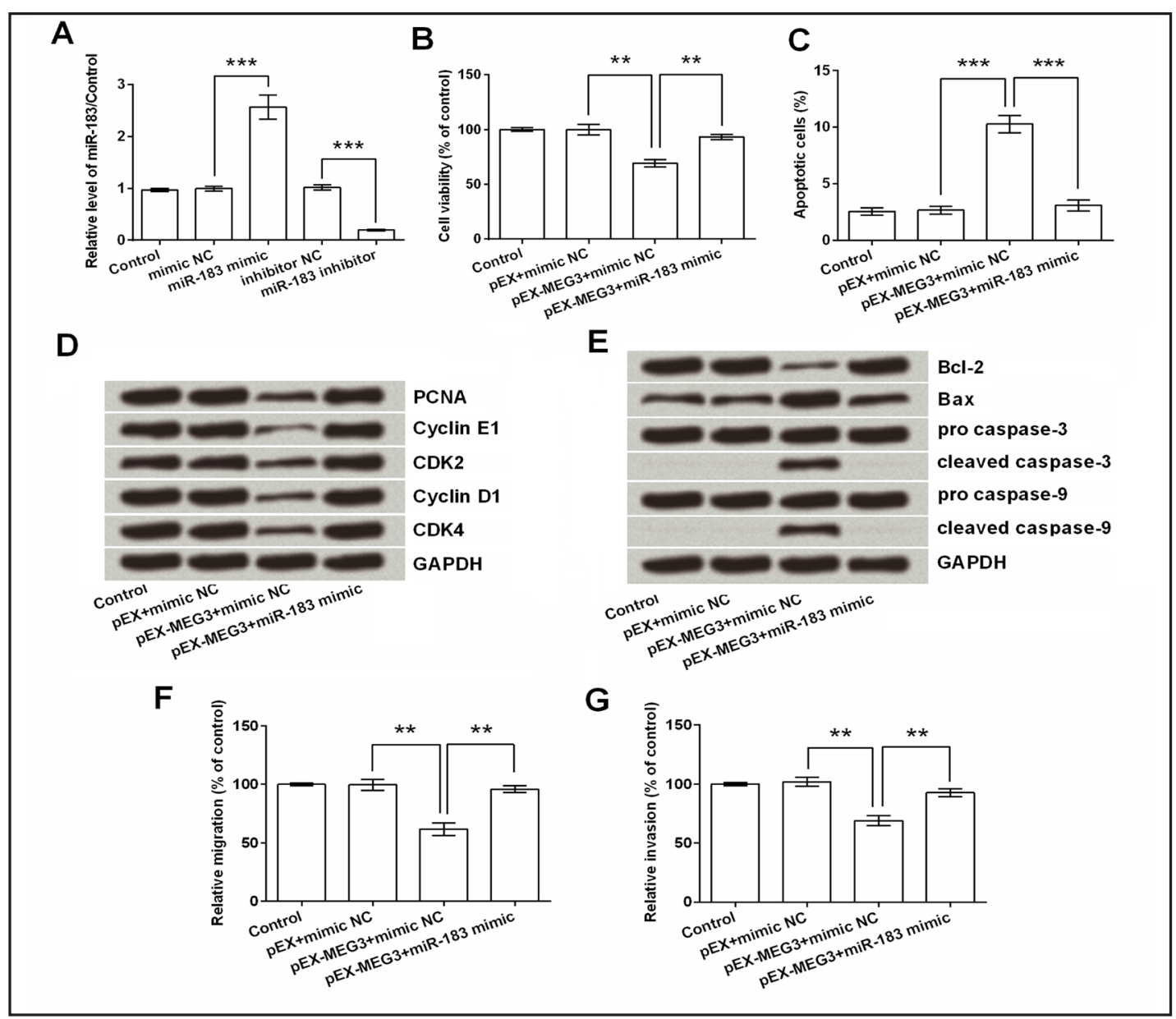

Fig. 4. miR-183 up-regulation abolished the anti-tumor functions of MEG3 in BON1 cells. (A) BON1 cells were transfected with miR-183 mimic, miR-183 inhibitor or their correspondingly negative controls for $48 \mathrm{~h}$. The expression of miR-183 in cells was then measured by qRT-PCR. BON1 cells were co-transfected with MEG3 expressing vector (pEX-MEG3) and miR-183 mimic for $48 \mathrm{~h}$. After another $48 \mathrm{~h}$ of incubation in the fresh medium, (B) cell viability, (C) apoptotic cells rate, (D) the protein expression of cell cycle related factors, (E) the protein expression of apoptosis associated factors, (F) cell migration, and (G) cell invasion were respectively assessed by typan blue staining, flow cytometry, western blotting, and transwell assay. Data represent the mean \pm SD of three independent experiments. ${ }^{* *} \mathrm{P}<0.01$, and ${ }^{* *} \mathrm{P}<0.01$ (ANOVA).

proteins were all down-regulated in BON1 silencing-cells, indicating p38/ERK/AKT and Wnt/ $\beta$-Catenin signaling pathways were both activated by BON1 overexpression.

\section{Discussion}

In this research, we found that MEG3 was low expressed in BON1 and QGP-1 cells. In addition, MEG3 overexpression significantly decreased BON1 cells viability, invasion, migration, but significantly induced apoptosis. These findings suggested that MEG3 exerted anti-tumor activities in pNETs. miR-183 was a direct target of MEG3, and miR-183 upregulation abolished the anti-growth and anti-metastasis effects of MEG3 overexpression on BON1 cells. Moreover, BRI3 was positively regulated by miR-183, and BRI3 exhibited a tumor-promoting role possibly via activation of p38/ERK/AKT and Wnt/ $\beta$-Catenin signaling pathways in BON1 cells.

\section{KARGER}


Fig. 5. BRI3 was a direct target of miR-183. (A) The expression level of BRI3 in human kidney epithelial cell line HEK293 and human pancreatic neuroendocrine tumor (pNET) cell line BON1 and QGP-1 were detected by qRT-PCR. (B-C) BON1 cells were transfected with miR-183 mimic, miR-183 inhibitor or their correspondingly negative controls for 48 h. Both the mRNA and protein levels
B

C

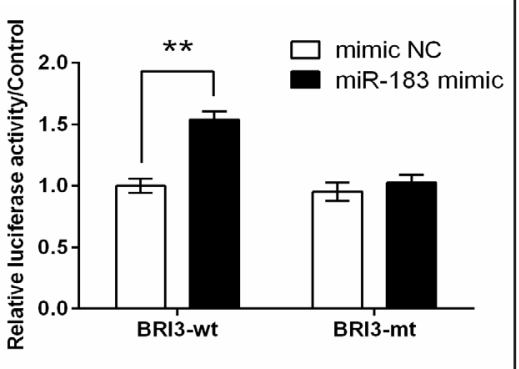

BRI3

GAPDH
D

of BRI3 in cells were measured by qRT-PCR and western blotting. (D) Dual-luciferase reporter assay was performed to detect whether BRI3 was a target of miR-183. Data represent the mean \pm SD of three independent experiments. ${ }^{* *} \mathrm{P}<0.01$, and ${ }^{* *} \mathrm{P}<0.01$ (ANOVA).

Fig. 6. BRI3 promoted BON1 cells growth and metastasis. BON1 cells were transfected with BRI3 expressing vector ( $\mathrm{pEX}$ BRI3), BRI3 targeted shRNA (shBRI3), or their negative controls (pEX and shNC) for 48 h. (A) The expression of BRI3 in cells was detected by qRT-PCR. After another $48 \mathrm{~h}$ of incubation in the fresh medium, (B) cell viability, (C) apoptotic

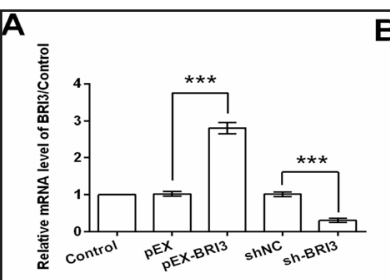

D

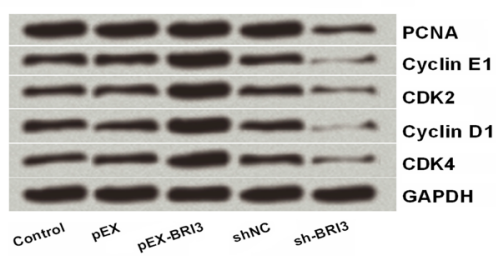

$\mathbf{F}$

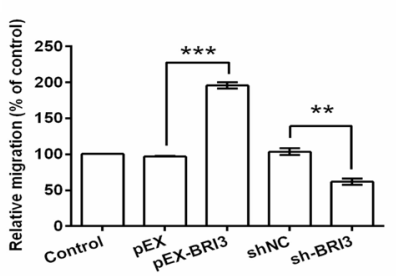

G
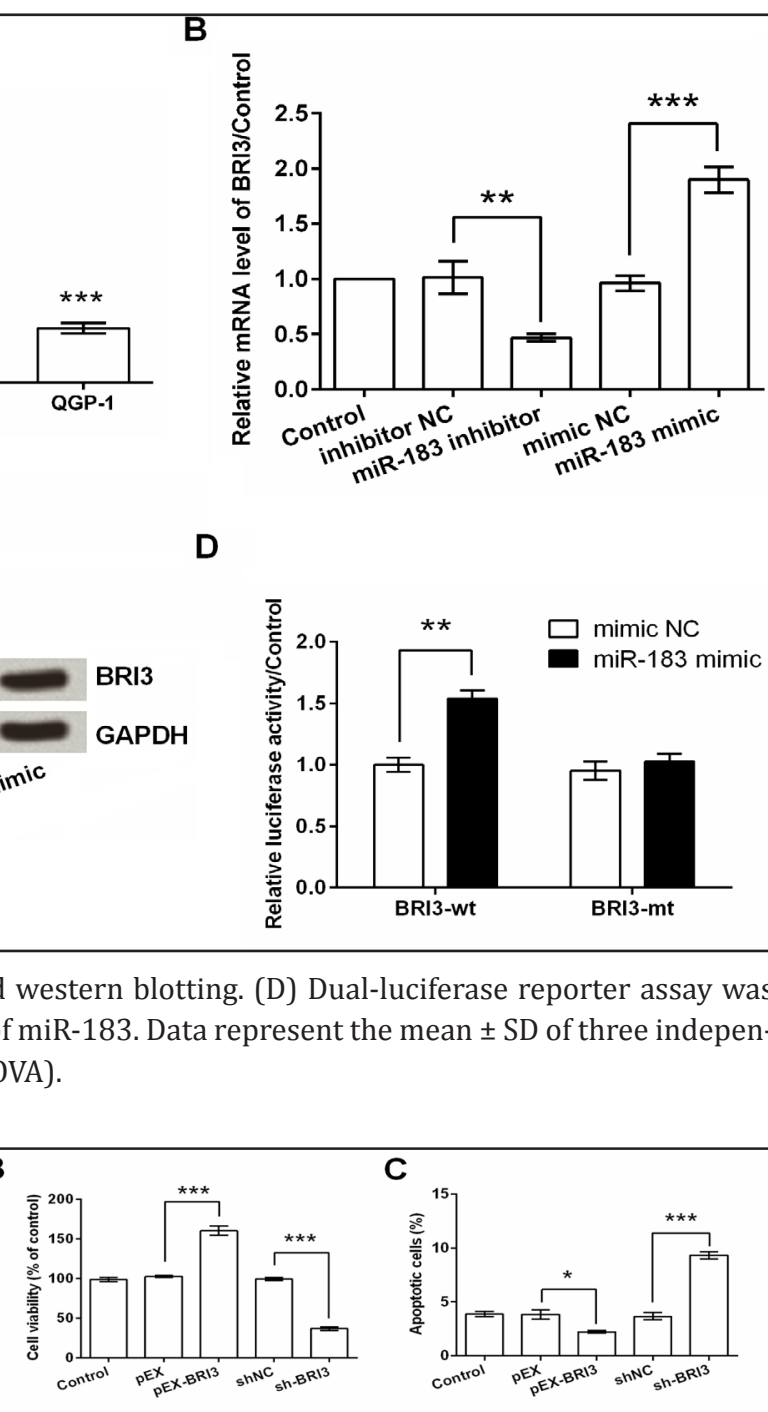

E
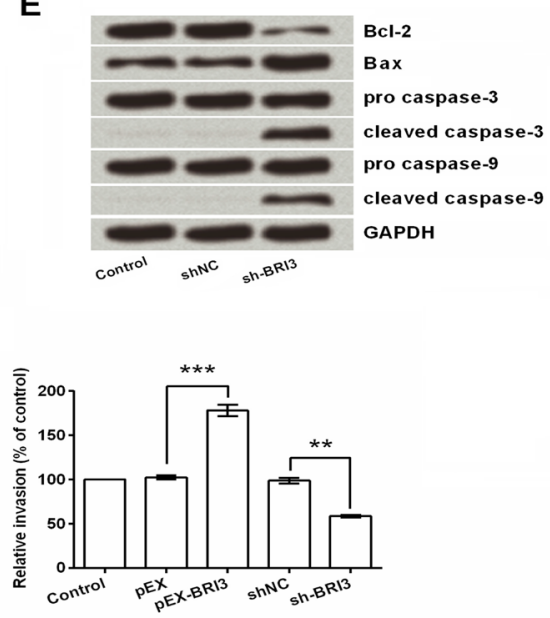

cells rate, (D) the protein expression of cell cycle related factors, (E) the protein expression of apoptosis associated factors, (F) cell migration, and (G) cell invasion were respectively assessed by typan blue staining, flow cytometry, western blotting, and transwell assay. Data represent the mean \pm SD of three independent experiments. * $\mathrm{P}<0.05$, ** $\mathrm{P}<0.01$, and ${ }^{* *} \mathrm{P}<0.01$ (ANOVA). 


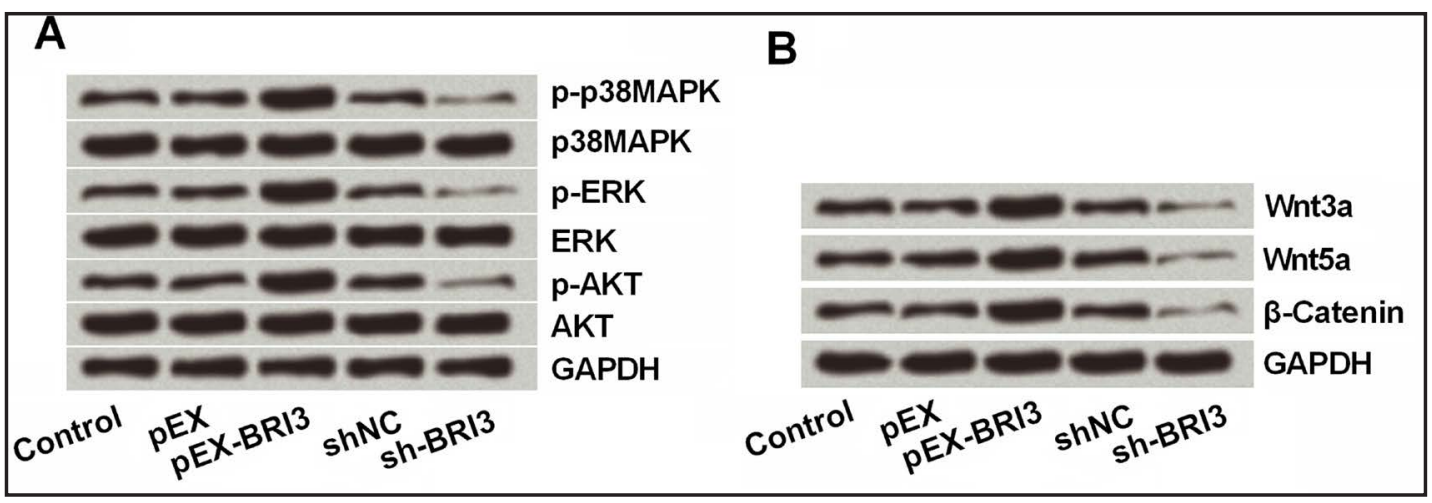

Fig. 7. BRI3 activated p38/ERK/AKT and Wnt/ $\beta$-Catenin signaling. BON1 cells were transfected with BRI3 expressing vector ( $p E X-B R I 3), B R I 3$ targeted shRNA (sh-BRI3), or their negative controls (pEX and shNC) for $48 \mathrm{~h}$. The protein expressions of main factors in (A) p38/ERK/AKT and (B) Wnt/ $\beta$-Catenin signaling were determined by western blot analysis.

MEG3 is a newly recognized IncRNA. Reduced expression of MEG3 has been found in variety of cancers tissues and cell lines [7-11]. In our current study, we have demonstrated that MEG3 was low expressed in BON1 and QGP-1 cells when compared to HEK293, CCL153, and EC-304 cell lines. This finding revealed that MEG3 might be implicated in the tumorigenesis and development of pNETs. Actually, the tumor suppressor properties of MEG3 have been found in multiple cancers. Re-constitution of MEG3 led to a decrease in cell proliferation and elevated apoptosis of lung cancer cells [23]. Another investigation demonstrated MEG3 suppressed breast cancer cells proliferation, colony formation, migration, and invasion capacities [24]. Further, MEG3 has been found to induce prostate cancer cells apoptosis via reducing the protein expression of Bcl-2, enhancing Bax, and activating caspase-3, as well as to induced cell cycle arrest in G0/G1 phase by suppressing Cyclin D1 [25]. MEG3 has also been reported as a tumor suppressor in pNET cells, because the overexpression of MEG3 in MIN6 (insulin-secreting mouse pNET cell line) blocked cell proliferation, delayed cell cycle progression, and reduced cell migration and invasion [26]. The findings in the present study were consistence with this previous study suggested the anti-tumor capacities of MEG3 in pNETs, as MEG3 overexpression suppressed BON1 cells viability, cell cycle progression, migration, invasion, and induced cell apoptosis.

To further explore the underling mechanisms via which MEG3 suppressed BON1 cells, we focused on the cross-regulation between miR-183 and MEG3, since ceRNA hypothesis is one of the theories that well explain the interactions between IncRNA and miRNA [15]. The pro-growth and anti-apoptosis role of miR-183 in several cancer cells have been described. For instance, miR-183 suppression reduced tongue squamous cell carcinoma SCC25 cells growth and colony formation, while the apoptosis percentage was significantly increased [27]. Ruan and his colleagues provided both in vitro and in vivo data suggested that knockdown of miR-183 significantly attenuated endometrial cancer cells growth and metastasis [28]. In our study, through dual-luciferase reporter assay we found that miR183 was a target of MEG3, and miR-183 overexpression abolished the anti-tumor effects of MEG3. Hence, we inferred that MEG3 suppressed BON1 cells growth and metastasis might be via interaction with miR-183.

BRI3 was originally cloned as a type II transmembrane protein of 267 amino acids [29]. It has been reported that BRI3 up-regulation results in the proliferation and migration of breast cancer cells [22]. Interestingly, we also show here that BRI3 has carcinogenic activity in pNETs, as BON1 cells growth and metastasis were promoted after BRI3 was overexpressed. Besides, by using dual-luciferase reporter assay we found BON1 was a direct target of miR183. Together with our findings that miR-183 was a target of MEG3, these data inspired us to hypothesize that MEG3 inhibits BON1 cells growth and metastasis via down-regulation of miR-183, and thus targeted BRI3. 


\section{Cellular Physiology Cell Physiol Biochem 2017;44:345-356 \begin{tabular}{l|l|l}
\hline DOI: 10.1159/000484906 & $\begin{array}{l}\text { C) } 2017 \text { The Author(s). Published by S. Karger AG, Basel } \\
\text { www.karger.com/cpb }\end{array}$
\end{tabular} \\ Zhang/Feng: the Role of MEG3 in Pancreatic Neuroendocrine Tumor}

p38MAPK and ERK are two important proteins in the MAPK pathway, and MAPK/AKT has been associated with multiple kind of cancers by modulating tumor cells proliferation, apoptosis, and metastasis [30-32]. Similar, Wnt/ $\beta$-Catenin has also been reported as a pivotal regulator of tumor cells metastasis [33-35]. Thus, to further explore the molecular mechanism via which BRI3 modulated BON1 cells, we monitored the protein levels of main factors in $\mathrm{p38}$ / ERK/AKT and Wnt/ $\beta$-Catenin signaling pathways in response to the BRI3 silence and BRI3 overexpression. After BRI3 silenced, both these two pathways were blocked, as p38MAPK, ERK, and AKT were deactivated, and Wnt3a, Wnt5a, and $\beta$-Catenin were down-regulated. These data suggested BRI3 exerted carcinogenic activity via regulation of p38/ERK/AKT and Wnt/ $\beta$-Catenin signaling. Our data were consistence with previous studies that by using serial analysis of gene expression, genome-wide microarray and promoter analysis, BRI3 has been identified as a target of Wnt/ $\beta$-catenin signaling [36]. Additionally, silencing of BRI3 expression clearly suppressed the phosphorylation of ERK, and consequently promoted the proliferation and migration of breast cancer cells [22].

There exist several limitations in the present study. Firstly, in this study we focused on the tumor-suppressive activities and the underling mechanisms of MEG3 on BON1 cells. The role of MEG3 in other cell types, especially primary pNET cells need to be revealed to crosscheck the effects of MEG3 in pNET. Secondly, this study lack of detection of MEG3, miR183 and BRI3 expressions in pNET tissues, which may better reveal the importance of them in pNET.

To sum up, this study demonstrated a tumor suppressive effect of MEG3 in BON1 cells that suppress tumor cells growth and metastasis. MEG3 may act as a ceRNA via sponging miR-183 and thus target BRI3. Our study revealed a novel regulatory mechanism that modulation of MEG3/miR-183/BRI3 axis may be pivotal in pNET.

\section{Acknowledgements}

The work received no funding support from any agency.

\section{Disclosure Statement}

There is no conflict of interests.

\section{References}

1 Mirica A, Badarau IA, Mirica R, Paun S, Paun DL: A rare case of metastasized non-functional pancreatic neuroendocrine tumor with a good long-term survival. J Med Life 2016;9:369-372.

-2 Jilesen AP, van Eijck CH, in’t Hof KH, van Dieren S, Gouma DJ, van Dijkum EJ: Postoperative Complications, In-Hospital Mortality and 5-Year Survival After Surgical Resection for Patients with a Pancreatic Neuroendocrine Tumor: A Systematic Review. World J Surg 2016;40:729-748.

-3 Sun J: Pancreatic neuroendocrine tumors. Intractable Rare Dis Res 2017;6:21-28.

4 Yang Y, Wang S, Li T: Altered long non-coding RNAs predict worse outcome in osteosarcoma patients: evidence from a meta-analysis. Oncotarget 2017;8:35234-35243.

-5 Lu Z, Li Y, Wang J, Che Y, Sun S, Huang J, Chen Z, He J: Long non-coding RNA NKILA inhibits migration and invasion of non-small cell lung cancer via NF-kappaB/Snail pathway. J Exp Clin Cancer Res 2017;36:54.

6 Zheng Y, Yang C, Tong S, Ding Y, Deng W, Song D, Xiao K: Genetic variation of long non-coding RNA TINCR contribute to the susceptibility and progression of colorectal cancer. Oncotarget 2017;8:33536-33543.

7 Sheng X, Li J, Yang L, Chen Z, Zhao Q, Tan L, Zhou Y, Li J: Promoter hypermethylation influences the suppressive role of maternally expressed 3, a long non-coding RNA, in the development of epithelial ovarian cancer. Oncol Rep 2014;32:277-285. 


\section{Cellular Physiology Cell Physiol Biochem 2017;44:345-356 \begin{tabular}{l|l} 
and Biochemistry Published online: November 13, 2017 & $\begin{array}{l}\text { (c) } 2017 \text { The Author(s). Published by S. Karger AG, Basel } \\
\text { www.karger.com/cpb }\end{array}$ \\
\hline POI.159/00048406
\end{tabular}}

Zhang/Feng: the Role of MEG3 in Pancreatic Neuroendocrine Tumor

8 Sun M, Xia R, Jin F, Xu T, Liu Z, De W, Liu X: Downregulated long noncoding RNA MEG3 is associated with poor prognosis and promotes cell proliferation in gastric cancer. Tumour Biol 2014;35:1065-1073.

-9 Yin DD, Liu ZJ, Zhang E, Kong R, Zhang ZH, Guo RH: Decreased expression of long noncoding RNA MEG3 affects cell proliferation and predicts a poor prognosis in patients with colorectal cancer. Tumour Biol 2015;36:4851-4859.

10 Zhang J, Lin Z, Gao Y, Yao T: Downregulation of long noncoding RNA MEG3 is associated with poor prognosis and promoter hypermethylation in cervical cancer. J Exp Clin Cancer Res 2017;36:5.

11 Tong GF, Qin N, Sun LW, Xu XL: Long Noncoding RNA MEG3 Suppresses Glioma Cell Proliferation, Migration, and Invasion By Acting As Competing Endogenous RNA of MiR-19a. Oncol Res 2017. Doi:10.3727/0965040 17x14886689179993

12 Tian P, Yan L: Inhibition of MicroRNA-149-5p Induces Apoptosis of Acute Myeloid Leukemia Cell Line THP1 by Targeting Fas Ligand (FASLG). Med Sci Monit 2016;22:5116-5123.

13 Cai H, Yao J, An Y, Chen X, Chen W, Wu D, Luo B, Yang Y, Jiang Y, Sun D, He X: LncRNA HOTAIR acts a competing endogenous RNA to control the expression of notch3 via sponging miR-613 in pancreatic cancer. Oncotarget 2017;8:32905-32917.

14 He JH, Li BX, Han ZP, Zou MX, Li W, Lv YB, Zhou JB, Cao MR, Li YG, Zhang JZ: Snail-activated long noncoding RNA PCA3 up-regulates PRKD3 expression by miR-1261 sponging, thereby promotes invasion and migration of prostate cancer cells. Tumor Biol 2016;1-14.

-15 Zhou X, Yuan P, Liu Q, Liu Z: LncRNA MEG3 Regulates Imatinib Resistance in Chronic Myeloid Leukemia via Suppressing MicroRNA-21. Biomol Ther (Seoul) 2017;25:490-496.

-16 Zhang J, Yao T, Wang Y, Yu J, Liu Y, Lin Z: Long noncoding RNA MEG3 is downregulated in cervical cancer and affects cell proliferation and apoptosis by regulating miR-21. Cancer Biol Ther 2016;17:104-113.

17 Yang NQ, Luo XJ, Zhang J, Wang GM, Guo JM: Crosstalk between Meg3 and miR-1297 regulates growth of testicular germ cell tumor through PTEN/PI3K/AKT pathway. Am J Transl Res 2016;8:1091-1099.

-18 Li Z, Jin C, Chen S, Zheng Y, Huang Y, Jia L, Ge W, Zhou Y: Long non-coding RNA MEG3 inhibits adipogenesis and promotes osteogenesis of human adipose-derived mesenchymal stem cells via miR-140-5p. Mol Cell Biochem 2017. Doi:10.1007/s11010-017-3015-Z

19 Bai J, Na H, Hua X, Wei Y, Ye T, Zhang Y, Jian G, Zeng W, Yan L, Tang Q: A retrospective study of NENs and miR-224 promotes apoptosis of BON-1 cells by targeting PCSK9 inhibition. Oncotarget 2017;8:6929-6939.

20 Livak KJ, Schmittgen TD: Analysis of relative gene expression data using real-time quantitative PCR and the 2(-Delta Delta C(T)) Method. Methods 2001;25:402-408.

21 Lima CR, Gomes CC, Santos MF: Role of microRNAs in endocrine cancer metastasis. Mol Cell Endocrinol 2017;456:62-75.

22 Guo DQ, Zhang H, Tan SJ, Gu YC: Nifedipine promotes the proliferation and migration of breast cancer cells. PLoS One 2014;9:e113649.

-23 Kruer TL, Dougherty SM, Reynolds L, Long E, de Silva T, Lockwood WW, Clem BF: Expression of the lncRNA Maternally Expressed Gene 3 (MEG3) Contributes to the Control of Lung Cancer Cell Proliferation by the Rb Pathway. PLoS One 2016;11:e0166363.

-24 Sun L, Li Y, Yang B: Downregulated long non-coding RNA MEG3 in breast cancer regulates proliferation, migration and invasion by depending on p53's transcriptional activity. Biochem Biophys Res Commun 2016;478:323-329.

25 Luo G, Wang M, Wu X, Tao D, Xiao X, Wang L, Min F, Zeng F, Jiang G: Long Non-Coding RNA MEG3 Inhibits Cell Proliferation and Induces Apoptosis in Prostate Cancer. Cell Physiol Biochem 2015;37:2209-2220.

-26 Modali SD, Parekh VI, Kebebew E, Agarwal SK: Epigenetic regulation of the lncRNA MEG3 and its target c-MET in pancreatic neuroendocrine tumors. Mol Endocrinol 2015;29:224-237.

27 Yan D, Cai X, Feng Y: miR-183 Modulates Cell Apoptosis and Proliferation in Tongue Squamous Cell Carcinoma SCC25 Cell Line. Oncol Res 2016;24:399-404.

28 Ruan H, Liang X, Zhao W, Ma L, Zhao Y: The effects of microRNA-183 promots cell proliferation and invasion by targeting MMP-9 in endometrial cancer. Biomed Pharmacother 2017;89:812-818.

29 Deleersnijder W, Hong G, Cortvrindt R, Poirier C, Tylzanowski P, Pittois K, Van Marck E, Merregaert J: Isolation of markers for chondro-osteogenic differentiation using cDNA library subtraction. Molecular cloning and characterization of a gene belonging to a novel multigene family of integral membrane proteins. J Biol Chem 1996;271:19475-19482. 


\section{Cellular Physiology Cell Physiol Biochem 2017;44:345-356 \begin{tabular}{ll|l} 
DOI: 10.1159/000484906 & O 2017 The Author(s). Published by S. Karger AG, Basel \\
www.kargercom/cph
\end{tabular} \\ Zhang/Feng: the Role of MEG3 in Pancreatic Neuroendocrine Tumor}

-30 Palanivel K, Kanimozhi V, Kadalmani B, Akbarsha MA: Verrucarin A induces apoptosis through ROSmediated EGFR/MAPK/Akt signaling pathways in MDA-MB-231 breast cancer cells. J Cell Biochem 2014;115:2022-2032.

-31 Sui JQ Xie KP, Zou W, Xie MJ: Emodin inhibits breast cancer cell proliferation through the ERalpha-MAPK/ Akt-cyclin D1/Bcl-2 signaling pathway. Asian Pac J Cancer Prev 2014;15:6247-6251.

32 Chen WN, Chen JY, Jiao BY, Lin WS, Wu YL, Liu LL, Lin X: Interaction of the hepatitis B spliced protein with cathepsin B promotes hepatoma cell migration and invasion. J Virol 2012;86:13533-13541.

33 Qin M, Zhang J, Xu C, Peng P, Tan L, Liu S, Huang J: Knockdown of NIK and IKKbeta-Binding Protein (NIBP) Reduces Colorectal Cancer Metastasis through Down-Regulation of the Canonical NF-kappaBeta Signaling Pathway and Suppression of MAPK Signaling Mediated through ERK and JNK. PLoS One 2017;12:e0170595.

34 Masui O, Ueda Y, Tsumura A, Koyanagi M, Hijikata M, Shimotohno K: RelA suppresses the Wnt/beta-catenin pathway without exerting trans-acting transcriptional ability. Int J Mol Med 2002;9:489-493.

35 Yang Y, Quan L, Ling Y: RBMS3 Inhibits the Proliferation and Metastasis of Breast Cancer Cells. Oncol Res 2017. Doi:10.3727/096504017x14871200709504

-36 Kavak E, Najafov A, Ozturk N, Seker T, Cavusoglu K, Aslan T, Duru AD, Saygili T, Hoxhaj G, Hiz MC, Unal DO, Birgul-Iyison N, Ozturk M, Koman A: Analysis of the Wnt/B-catenin/TCF4 pathway using SAGE, genomewide microarray and promoter analysis: Identification of BRI3 and HSF2 as novel targets. Cell Signal 2010;22:1523-1535. 